\title{
Parallel Control of Shunt Active Power Filters in Capacity Proportion Frequency Allocation Mode
}

\author{
Shuquan Zhang ${ }^{\dagger}$, Ke Dai*, Bin Xie*, and Yong Kang* \\ $\dagger^{\dagger *}$ Dept. of Electrical and Electronics Eng., Huazhong University of Science and Technology, Wuhan, China
}

\begin{abstract}
A parallel control strategy in capacity proportion frequency allocation mode for shunt active power filters (APFs) is proposed to overcome some of the difficulties in high power applications. To improve the compensation accuracy and overall system stability, an improved selective harmonic current control based on multiple synchronous rotating reference coordinates is presented in a single APF unit, which approximately implements zero steady-state error compensation. The combined decoupling strategy is proposed and theoretically analyzed to simplify selective harmonic current control. Improved selective harmonic current control forms the basis for multi-APF parallel operation. Therefore, a parallel control strategy is proposed to realize a proper optimization so that the APFs with a larger capacity compensate more harmonic current and the ones with a smaller capacity compensate less harmonic current, which is very practical for accurate harmonic current compensation and stable grid operation in high power applications. This is verified by experimental results. The total harmonic distortion (THD) is reduced from $29 \%$ to $2.7 \%$ for a typical uncontrolled rectifier load with a resistor and an inductor in a laboratory platform.
\end{abstract}

Key Words: Active power filter (APF), Frequency allocation, Parallel, Selective harmonic, Zero steady-state error

\section{INTRODUCTION}

Harmonic and reactive currents caused by the continuous proliferation of nonlinear loads such as high-power diodes/thyristors, rectifiers, cycloconverters, and arc furnaces in industrial, commercial and residential applications are growing problems. Harmonic and reactive currents excite more and more attentions since they increase power losses, deteriorate the quality of current and voltage waveforms, produce extra neutral currents, mislead metering devices, and may cause resonances and interferences. Various types of harmonic and reactive power compensators have been researched and developed [1]-[4].

Active power filters (APFs) are power electronic devices used mainly for harmonic current compensation, and have become a hot topic in the last decade. Compared to traditional LC passive filters, APFs have many advantages such as small size and light weight, low power losses, tracking of the power system frequency change, fast dynamic response to load changes and reduction of resonant problems. In addition, APFs can provide other conditioning functions such as reactive power control, load balancing and flicker mitigation. With an increase in nonlinear load capacity, the load currents that need to be compensated may exceed the rated power of a single APF unit. In this case, an APF with a large capacity or the parallel operation of several APFs provides solutions [5]. This paper is focused on the parallel operation of APFs to improve

\footnotetext{
Manuscript received Nov. 26, 2009; revised May 15, 2010

†Corresponding Author: Shuquan_zhang@qq.com

Tel: +86-027-87543071, Fax: +86-027-87543658, HUST

* Dept. of Electrical and Electronics Eng., HUST, China
}

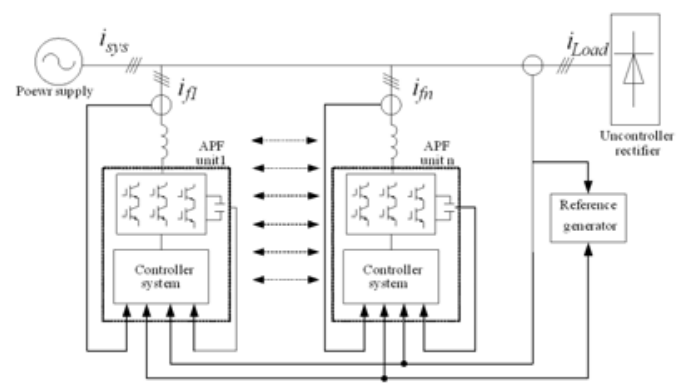

Fig. 1. The topology of APFs parallel system.

compensation accuracy and overall system stability.

Recent research on the parallel operation of APFs has concentrated on operation safety and parallel topology analysis. The current limited control strategy proposed in [6] and [7] guarantees that the output currents of each single APF unit in a parallel system are within the range of their respective capacity. Some possible parallel operation topologies were studied in [8] and [9]. The topology shown in Fig.1 is one of the best choices in terms of parallel system stability and flexibility in high power applications, which was attacked by its lower compensation accuracy. All APF units shown in Fig.1 adopt the feed forward control. Therefore, good stability is realized [10], and the convenience of installation and utilization makes the topology more acceptable to customers. To significantly enhance the compensation accuracy, improved selective harmonic current control is applied to a single APF unit which forms the basis of multi-APF parallel operation, which theoretically realizes harmonic currents compensation 
with zero steady-state error [11]-[15].

In this paper, a selective harmonic current control based on multiple synchronous rotating reference coordinates is presented and a combined decoupling strategy is further proposed to simplify the selective harmonic current control. Due to the detection and control in the respective frame, a proportional integral (PI) controller in the harmonic frame is one of the best choices when compared to the controllers adopted in the existing selective harmonic current control strategies such as the generalized integrator [12], the repetitive-based controller [13] and the proportion resonance controller [15]. However, a PI controller in the harmonic frame requires extensive computation resources from the control chip. The proposed parallel control strategy in capacity proportion frequency allocation mode not only overcomes the aforementioned difficulty but also significantly improves the compensation accuracy and the overall system stability in high power applications. The proposed parallel control strategy has been validated for some special industrial applications which have high requirements for harmonic current compensation accuracy.

This paper is organized as follows: In Section II, the block diagram of a single APF unit in a parallel system is described, and a selective harmonic current control adopting the combined decoupling strategy is proposed. In Section III, the parallel control strategy in capacity proportion frequency allocation mode is proposed and explicitly explained. The experimental results for the improved selective harmonic current control and the parallel control strategy are shown in Section IV. Finally, a discussion and conclusion are presented in Section V.

\section{IMPROVED SELECTIVE HARMONIC CURRENT CONTROL FOR A SINGLE APF UNIT}

The main objective of an APF is to generate currents which are the inverse of the load harmonic currents to make the grid currents sinusoidal and the power factor of a power system unity. The basic block diagram of a single APF unit with a nonlinear load connected to a three-phase three-wire power system is shown in Fig.2.

The part in the dashed box is the control system of a single APF unit that compensates each order of harmonic current. The control system includes a direct current (DC) link voltage control loop, a fundamental current control loop, and multiple selective harmonic current control loops. Note thatonly a selective $\mathrm{n}$-th order harmonic current control loop is shown in Fig.2. Multiple loops will be explained in detail in Fig.4. The reference voltage of the APF unit is the superposition of the fundamental current controller output and all the harmonic current controllers outputs, and it is realized by a space vector pulse width modulation (SVPWM) unit.

The DC link voltage controller is a PI control unit, which receives the reference voltage $U_{d c}^{*}$ and the measured DC link voltage $U_{d c}$, and outputs the fundamental active reference current. The fundamental current controller receives the fundamental active current references and the measured output current of APF unit in the fundamental frame to keep the DC link voltage stable at the reference voltage $U_{d c}^{*}$.
The selective harmonic current controller receives the reference signals from the corresponding selective harmonic current extraction unit, and controls the output currents of the APF unit to track the corresponding current components of the nonlinear loads, which is shown as the upper part of Fig.4. In this paper, we are only concerned with selective harmonic current detection and control.

\section{A. Mathematical Model of an APF Unit}

The configuration of a single APF unit is shown in Fig.3. $S_{a 1}, S_{a 2}, S_{b 1}, S_{b 2}, S_{c 1}, S_{c 2}$ are the switches of the three bridge arms, $L$ is the sum of the inner inductor $L_{1}$ and the outer inductor $L_{2} . R$ is the sum of the inductor internal resistance and the equivalent resistance caused by the dead band effect of the upper and lower switchs of each phase. $d c$ is the DC link capacitor which plays the role of storage energy. $R_{L}$ expresses the load effect which is drawn by the power loss of the APF.

$$
\left\{\begin{array}{l}
\frac{d i_{d n}}{d t}=-\frac{R}{L} i_{d n}+n \omega i_{q}-\frac{S_{d n}}{L_{q_{q n}}} U_{d c} \\
\frac{d t}{d t}=-\frac{R}{L} i_{q n}-n \omega i_{d n}-\frac{S_{q n}}{L} U_{d c} \\
\frac{d U_{d c}}{d t}=\frac{3 S_{d n}}{2 C_{d c}} i_{d n}+\frac{3 S_{q n}}{2 C_{d c}} i_{q n}-\frac{1}{C_{d c}} i_{d c 2}
\end{array} .\right.
$$

The mathematical model of a single APF unit is similar to the model of a PWM rectifier, which is presented along with the power system voltage disturbance and the output inductor current coupling in the fundamental frame [14]. In this paper, the mathematical models of the positive and negative sequence harmonic synchronous rotating coordinates are built as equation (1), where the power system voltage is neglected because the harmonic voltage component is very small. The model (1) reveals that the mathematical models of the positive and negative sequence harmonic synchronous rotating coordinates have the same mathematical form.

In equation (1), $n$ is the harmonic order. The mathematical relation can be deduced by the Park transformer. $T_{a b c-d q n}$ is one of the equations (2) or (3). Equation (2) is the $n$-th positive harmonic transformer and equation (3) is the $n$-th negative harmonic transformer from the three-phase stationary coordinate to the corresponding positive or negative harmonic synchronous rotating coordinates.

$$
\begin{aligned}
& T_{a b c-d q n}^{P}=\frac{2}{3}\left[\begin{array}{ccc}
\cos n \omega t & \cos \left(n \omega t-\frac{2 \pi}{3}\right) & \cos \left(n \omega t+\frac{2 \pi}{3}\right) \\
-\sin n \omega t & -\sin \left(n \omega t-\frac{2 \pi}{3}\right) & -\sin \left(n \omega t+\frac{2 \pi}{3}\right)
\end{array}\right] \\
& T_{a b c-d q n}^{N}=\frac{2}{3}\left[\begin{array}{ccc}
\cos n \omega t & \cos \left(n \omega t+\frac{2 \pi}{3}\right) & \cos \left(n \omega t-\frac{2 \pi}{3}\right) \\
-\sin n \omega t & -\sin \left(n \omega t+\frac{2 \pi}{3}\right) & -\sin \left(n \omega t+\frac{2 \pi}{3}\right)
\end{array}\right]
\end{aligned}
$$

\section{B. Selective Harmonic Currents Detection and Control}

The detection of selective harmonic currents in a synchronous rotating frame is simplified from the theory of synchronous rotating transformation. The three-phase harmonic currents in a stationary frame are alternating current (AC) components. The three-phase $\mathrm{n}$-th order harmonic currents $i_{a h n}, i_{b h n}$ and $i_{c h n}$ are transformed to the DC components $i_{d h n}$ and $i_{q h n}$ in the corresponding n-th harmonic synchronous rotating coordinate.

More specifically, the selected three-phase n-th order harmonic currents of the load currents are transformed to DC 


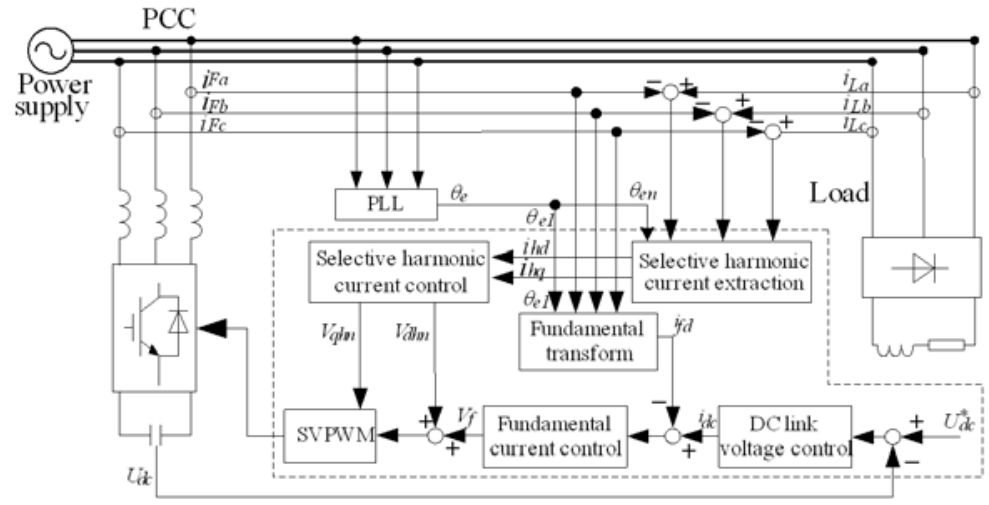

Fig. 2. Block diagram of APF unit.

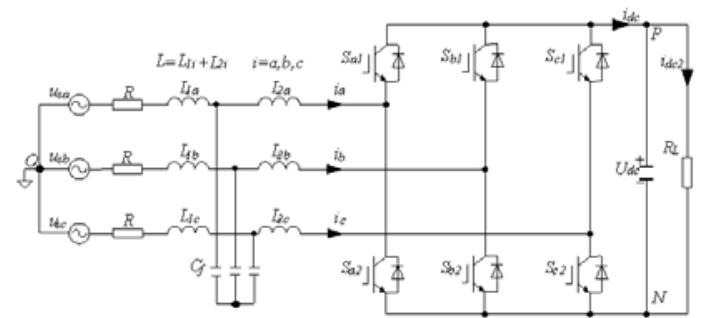

Fig. 3. The configuration of APF.

components on $\mathrm{d}$ axis and $\mathrm{q}$ axis, while the other non-selected harmonic currents and the three-phase fundamental currents are still AC components in the $\mathrm{n}$-th order harmonic synchronous rotating coordinate, whose frequency is an integral multiple of the power system frequency, and the average value in a period is zero. Then the transformed DC components can be extracted by low pass filters (LPFs) on the $\mathrm{d}$ axis and the $\mathrm{q}$ axis. In this paper, a moving average LPF is used to improve the dynamic response performance and the computational accuracy [16], whose $\mathrm{Z}$ domain transfer function is shown as equation (4).

$$
i_{d h n}(Z)=\frac{1}{Z}\left(\frac{Z}{Z-1}-\frac{Z}{Z-1} Z^{-N}\right) i_{d n}(Z)
$$

where $N$ is the number of samplings per power frequency period, and is set to 200 .

As mentioned above, the DC components $i_{d h n}$ and $i_{q h n}$ on the $\mathrm{d}$ axis and the $\mathrm{q}$ axis in the harmonic frame are correspondences with the three-phase $\mathrm{n}$-th order harmonic currents $i_{a h n}, i_{b h n}$ and $i_{c h n}$ in the three-phase stationary frame. Therefore, the detection of the selected $n$-th order harmonic currents of each phase is achieved. When multiple selected order harmonic currents need to be compensated, multiple corresponding extraction units are used and easily connected in parallel and conveniently realized in the program of a control chip. The detection method of the selective harmonic currents is shown in Fig.4.

Compared to the nonselective harmonic current control, the selective harmonic current control has several advantages in terms of flexibility and stability.

1) When the load harmonic currents are larger than the rated currents of the APF unit, the control system has the ability to select the most harmful harmonic currents to compensate for the purpose of overload protection.

2) In case of hybrid APFs, the LC passive filters and the APFs parallel connect to the power system. The former restrict the fixed order harmonic currents and the latter compensate the remaining harmonic currents. Under these circumstances, selective harmonic current control makes for a better utilization ratio of APF capacity and overall system stability.

3) The output inductor parameters may change with different frequencies of the output current. For selective harmonic current control, the selected harmonic currents are detected and separately controlled in their own corresponding frames. Changes in the inductor parameters and different delay times can be taken into account more easily with individual controllers since the selected frequencies are respectively designed, which can improve the overall system stability.

As described above, the selective three-phase harmonic currents are transformed into DC components, while the other non-selected harmonic currents and the fundamental currents are still $\mathrm{AC}$ components in their respective harmonic frames. The DC components can be extracted by a moving average LPF as in equation (4). A PI controller can track the DC components with zero steady-state error, and have good performance in terms of tracking accuracy, dynamic response and project practicality. Therefore, the PI controllers in the harmonic frames are adopted in this paper. As mentioned above, the DC components $i_{d h n}$ and $i_{q h n}$ on the $\mathrm{d}$ axis and the $\mathrm{q}$ axis in the selective $\mathrm{n}$-th order harmonic frame are correspondences with the selective three-phase harmonic currents by the Park transformer. Therefore, the output currents can track the three-phase selective harmonic currents by controlling the DC components $i_{d h n}$ and $i_{q h n}$ in the harmonic frame. The complete harmonic current controllers are achieved as the superposition of all of the harmonic current PI controllers, which is shown in Fig.4.

Due to detection and control in its respective frame, the PI controller in the harmonic frame is one of the best choices when compared to the controllers adopted in the existing selective harmonic current control strategies such as the generalized 


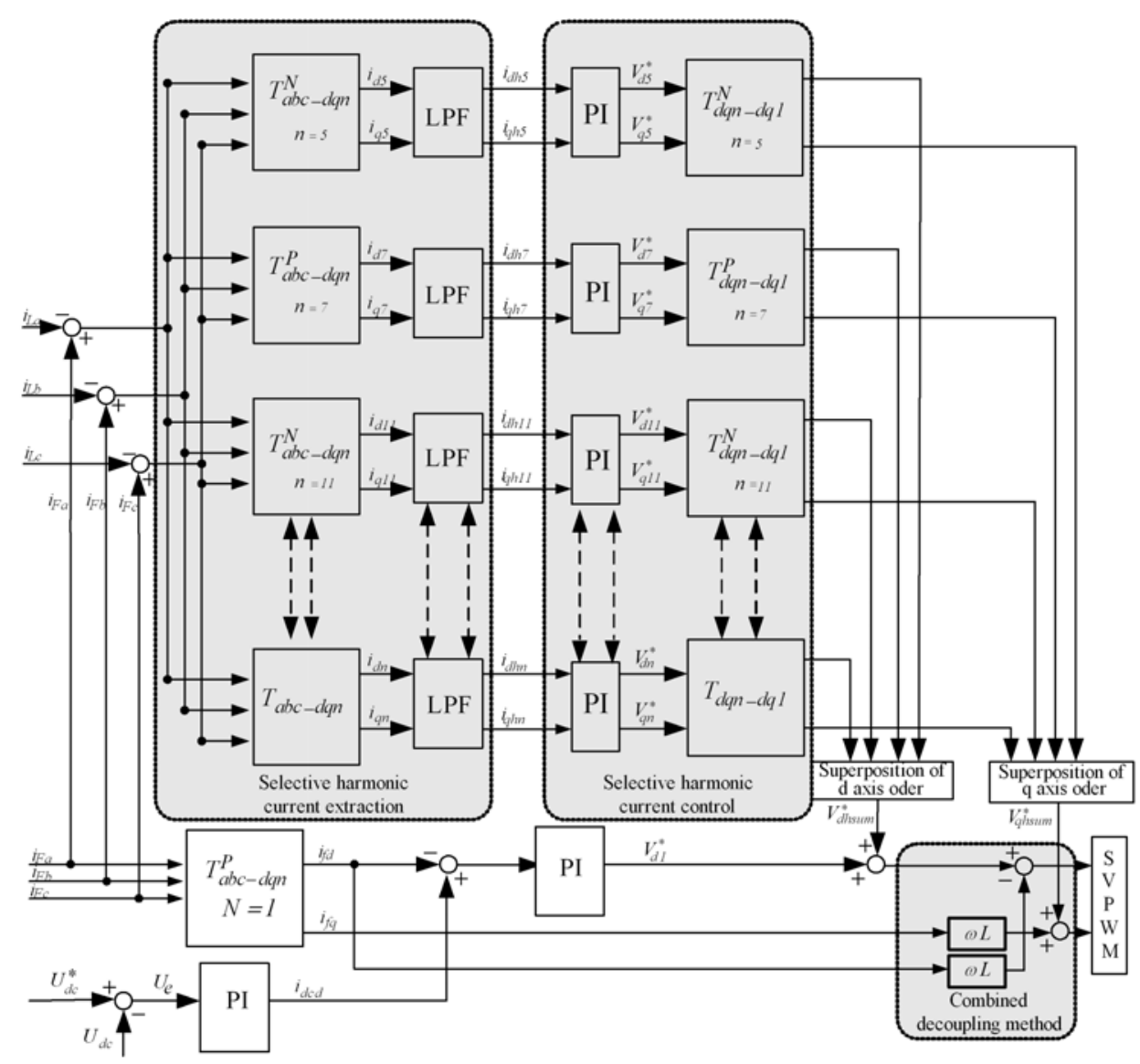

Fig. 4. Control system of APF unit.

integrator, the repetitive-based controller and the proportional resonant controller.

\section{Combined Decoupling Strategy}

SVPWM is realized in the fundamental frame and selective harmonic detection and control are in the harmonic frame. Therefore, the selective harmonic current control outputs need to be transformed to the fundamental frame. The transformer is equation (5) or (6) corresponding to the positive sequence harmonic or the negative sequence harmonic currents.

As we can see in equation (1), the $\mathrm{d}$ axis and the $\mathrm{q}$ axis currents have the coupling effect, which increases the control complexity. The traditional decoupling methods are implemented in their respective harmonic frames. As a result, control is complicated since multiple decoupling units are used. The combined decoupling strategy is proposed and shown in Fig.4. In this method, all the decoupling is achieved in the fundamental frame. Thus, the number of decoupling units is significantly reduced since only one decoupling unit is used. In order to prove the equivalent effect of the aforementioned combined decoupling strategy, transformation from the harmonic frame to the fundamental frame is needed.

For the positive sequence harmonic currents, the transformer from the harmonic coordinate to the fundamental coordinate is shown as equation (5).

$$
\begin{aligned}
& T_{d q n-d q 1}^{P}=T_{d q n-a b c}^{P} \times\left[T_{a b c-d q 1}\right]^{T} \\
& T_{d q n-d q 1}^{P}=\left[\begin{array}{cc}
\cos ((n-1) \omega t) & \sin ((n-1) \omega t) \\
-\sin ((n-1) \omega t) & \cos ((n-1) \omega t)
\end{array}\right] .
\end{aligned}
$$

For the negative sequence harmonic currents, the corresponding transformation is shown as equation (6).

$$
\begin{aligned}
& T_{d q n-d q 1}^{N}=T_{d q n-a b c}^{N} \times\left[T_{a b c-d q 1}\right]^{T} \\
& T_{d q n-d q 1}^{N}=\left[\begin{array}{cc}
\cos ((n+1) \omega t) & -\sin ((n+1) \omega t) \\
-\sin ((n+1) \omega t) & -\cos ((n+1) \omega t)
\end{array}\right] .
\end{aligned}
$$

Taking the $\mathrm{d}$ axis as an example, the mathematical model in the harmonic coordinate is shown as equation (7).

$$
\frac{d i_{d n}}{d t}=-\frac{R}{L} i_{d n}+n \omega i_{q n}-\frac{S_{d n}}{L} U_{d c}
$$

The above mathematical model can be transformed to the fundamental frame by equation (5) or (6), which is shown as equation (8).

$$
\frac{d i_{d 1 n}}{d t}=-\frac{R}{L} i_{d 1 n}+\omega i_{q 1 n}-\frac{S_{d 1 n}}{L} U_{d c} .
$$

Comparing equation (7) with equation (8), $i_{q n}$ in equation (7) is a DC component while $i_{q 1 n}$ in equation (8) is an AC 
component, what is more, $n \omega i_{q n}$ is equivalent to $n \omega i_{q 1 n}$. The conclusion is derived that the coupling parameter $n \omega L$ is different in different order harmonic coordinates, but they have the same value in the fundamental frame, which means that the traditional decoupling method and the proposed combined decoupling strategy have the same effect.

The improved selective harmonic current control is applied to a single APF unit in a parallel system. Although the control has good characteristics such as compensation accuracy, dynamic response and good stability, it requires extensive computation resources from the control chip, which limits its application to situations where many different order harmonic currents need to be compensated.

With an increase in non-linear load capacity, the harmonic current that needs to be compensated may exceed the rated power of a single APF unit. In this case, an APF unit with a large capacity or the parallel operation of several APFs provides solutions. The former is limited by the industry device level and existing technology. In terms of flexibility, redundancy and credibility, the latter is still a mainstream solution in the applications of low and medium voltage power systems. In order to improve the stability and compensation accuracy, a novel parallel control strategy is proposed in this paper.

\section{PARALLEL CONTROL OF APFS IN CAPACITY PROPORTION FREQUENCY ALLOCATION MODE}

In general, two types of harmonic compensation loops are applied in the control of APFs. One is a feedback loop and the other is a feedforward loop [8]. The feedback loop has better compensation accuracy compared to the feedforward loop. However, it may become unstable especially under unknown grid conditions. Furthermore, the installation and testing is inconvenient for end users, so it is not especially suited to industrial applications. On the other hand, the feedforward loop has good stability and straightforward installation, but the compensation accuracy is lower. Comparatively speaking, the feedforward loop is a better choice in industrial applications.

This paper focuses on improving the compensation accuracy of APFs in parallel systems adopting a feedforward loop, in order to satisfy situations where there is a high requirement for the reduction of every single order harmonic current distortion and total harmonic current distortion (THD). The parallel control of APFs in capacity proportion frequency allocation mode based on the improved selective harmonic current control is proposed to overcome the aforementioned difficulties in high power applications. A proper optimization based on APFs' capacities and load current spectrums is realized so that the APFs with a larger capacity compensate more harmonic currents and the ones with a smaller capacity compensate less harmonic currents. Simultaneously, zero steady-state error compensation is obtained. The proposed parallel control significantly improves the compensation accuracy and the stability of the overall system in high power applications.

The output inductor parameters change with the output current's frequency as mentioned above. A single APF unit outputs a single order or several adjacent order harmonic

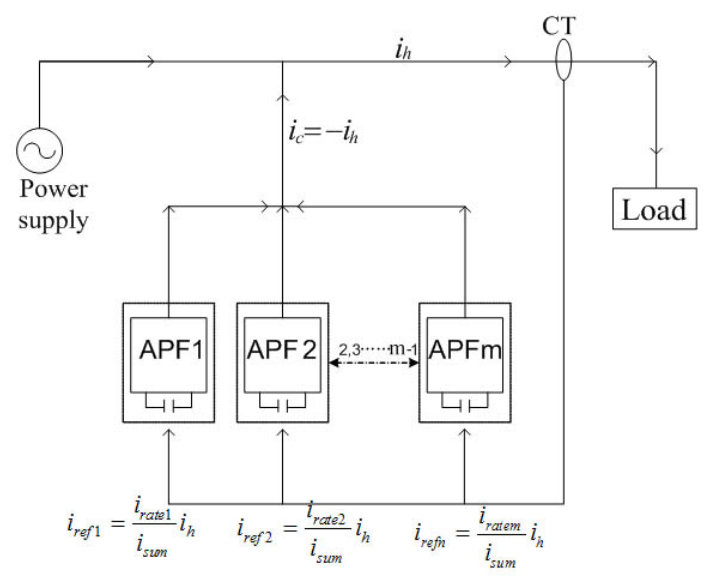

Fig. 5. Distributed parallel control of APFs.

currents, which improves the stability of the parallel system. When multiple selective harmonic currents need to be compensated, the multiple corresponding PI controllers of a single APF unit are used, which requires the DSP chip to do a large computation. According to the proposed parallel strategy, an APF compensates a single order or several adjacent order harmonic currents, which relieves the burden on the chip. Furthermore, it can be successfully applied in high power applications to improve the compensation accuracy of the APFs in a parallel system. The proposed strategy design will be described in more detail hereinafter.

As shown in Fig.5, $\mathrm{m}$ is the number of the APFs in a parallel system, $i_{h}$ is the total effective value of the load harmonic currents, $i_{\text {ratej }}(\mathrm{j}=1,2, \mathrm{~m})$ is the rated current of each single APF unit and $i_{r e f j}(\mathrm{j}=1,2, \mathrm{~m})$ is the corresponding reference current. Then the parallel system rated current value is shown as equation (9).

$$
\begin{aligned}
& i_{\text {sum }}^{m}=i_{\text {rate } 1}+i_{\text {rate } 2}+i_{\text {rate } 3}+\cdots \cdots+i_{\text {ratem }} \\
& =\sum_{j=1}^{m} i_{\text {ratej }} .
\end{aligned}
$$

The number of the APFs in a parallel system is determined by the mathematical relation between the total effective value of the load harmonic currents and the total rated currents of the APFs to be put into operation as shown in equation (10), which improves the utilization ratio and is beneficial for decreasing the overall system power loss.

$$
\sum_{j=1}^{m-1} i_{\text {ratej }} \leq i_{h} \leq \sum_{j=1}^{m} i_{\text {ratej }}
$$

Then, the order of the harmonic current to be compensated by a single APF should be clear according to the parallel strategy proposed. Each single order harmonic current's effective value can be calculated by the recursive Discrete Fourier Transform (DFT) within a period [17]. The THD and each single order harmonic current distortion can be derived according to the definition of the harmonic current distortion. Namely, the proportion of each single order harmonic current in the total harmonic current is determined. Correspondingly, 
the proportion of the capacity for each single APF unit in the parallel system is easily obtained. Therefore, the specific order harmonic currents to be compensated by the corresponding APF units with different capacities can be obtained according to the two proportion relation obtained above, which is shown as (11).

$$
\frac{\sum_{j \leq m} i_{\text {ratej }}}{i_{\text {sum }}} \geq \frac{\sum_{k<50} i_{k t h}}{i_{h}} .
$$

Usually, APFs are required to compensate harmonic currents within the fiftieth order. In equation (11), $\mathrm{k}$ is the order of the harmonic currents that need to be compensated, $k<50$ means the order of the compensated harmonic current is less than 50 and $\sum_{k<50} i_{k t h}$ is the effective value of a single order harmonic current or the sum of the effective values of several adjacent order harmonic currents.

For a single APF unit APFj, if the harmonic currents that need to be compensated are larger than the rated current of this APF unit, then is the effective value of a single order harmonic current within the range of the APFj capacity. Thus, $\sum_{k<50} i_{k t h}$ in equation (11) can be substituted by $i_{k t h}$, and $\sum_{j \leq m} i_{\text {ratej }}$ can be substituted by $i_{k t h}, i_{\text {ratej }} \leq i_{k t h}$. Since the rated current of the single APF unit is insufficient for the single order harmonic currents, the other APFs with the proper capacity are required to work together with APFj to compensate the order harmonic currents.

Conversely, if the capacity of APFj exceeds the single order harmonic current, $\mathrm{APFj}$ can compensate the other adjacent order harmonic currents according to the capacity proportion frequency allocation compensation strategy.

The proposed strategy has achieved success in some applications where compensated currents with a high quality waveform are required. Dynamic change of the load harmonic spectrum components is small in these typical situations. The proportion of each single order harmonic current in the load current has little change even if the load currents change fast, which makes the computation of change in the proposed parallel control small. Further research will be conducted when the load harmonic spectrum components have fast dynamic changes.

\section{EXPERIMENT}

TABLE I MAIN PARAMETERS OF APF UNIT

\begin{tabular}{|l|c|}
\hline Capacity & $66 \mathrm{kVA}$ \\
Output inductor & $0.35 \mathrm{mH}$ \\
DC link capacitor & $20 \mathrm{mF}$ \\
DC link voltage & $750 \mathrm{~V}$ \\
Switching frequency & $9.6 \mathrm{kHz}$ \\
Effective value of phase voltage & $220 \mathrm{~V}$ \\
Frequency of the power system & $50 \mathrm{~Hz}$ \\
\hline
\end{tabular}

The experimental results are presented in two parts: firstly, the improved selective harmonic current control is verified by compensating the fifth order harmonic current and the seventh order harmonic current separately, and then, the proposed parallel control strategy is verified by two APFs in parallel

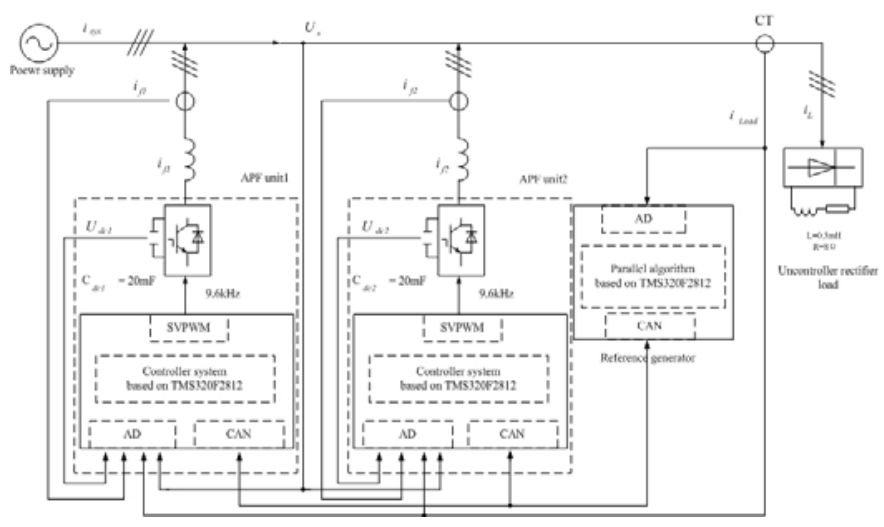

Fig. 6. General diagram of laboratory platform.

operation on the laboratory platform. Two APFs with the same capacity are adopted due to the conditional restrictions of the laboratory

\section{A. Implementation}

The proposed parallel control strategy is tested in the laboratory platform shown in Fig.6, which is composed of two APF units, a reference generator and a nonlinear uncontrolled rectifier load with a resistor and an inductor. The load currents are detected by the current transformers (CTs), and signals indicative of the load currents are provided to the reference generator and each single APF unit. The APF units communicate with the reference generator based on the CAN bus.

The reference generator receives the information on the APF units such as the unit number, the capacity and the working condition. Then it carries out the proposed parallel control algorithm to determine the reference instructions so that each single order or several adjacent order harmonic currents can be compensated by specific APF units. The APF units receive the reference instructions from reference generator and execute the improved selective harmonic current control to output the corresponding harmonic currents to make the power system currents $i_{\text {sys }}$ sinusoidal and the power factor of the power system unity.

The main electrical parameters of a single APF unit are listed in Table 1. The load is a typical three-phase uncontrolled rectifier with a resistor and an inductor. The values of the inductor and the resistor in the DC link are respectively $0.5 \mathrm{mH}$ and $8 \Omega$.

\section{B. Experiment results}

The load current waveform of one phase and its magnitude spectrum are shown in Fig.7. We can see that, the load current is seriously distorted due to the nonlinear load when the APF units do not operate. In this situation, the load current is the power system current. According to the spectrum analysis, the THD is $29 \%$, the fifth order harmonic current distortion $\left(5^{\text {th }}\right)$ is $23.27 \%$, the $7^{\text {th }}$ is $10.43 \%$, the $11^{\text {th }}$ is $9.25 \%$, the $13^{\text {th }}$ is $5.20 \%$, the $17^{\text {th }}$ is $5.34 \%$ and the other order harmonic current distortions are less than $3.5 \%$. The low frequency harmonic current distortion is especially serious. 


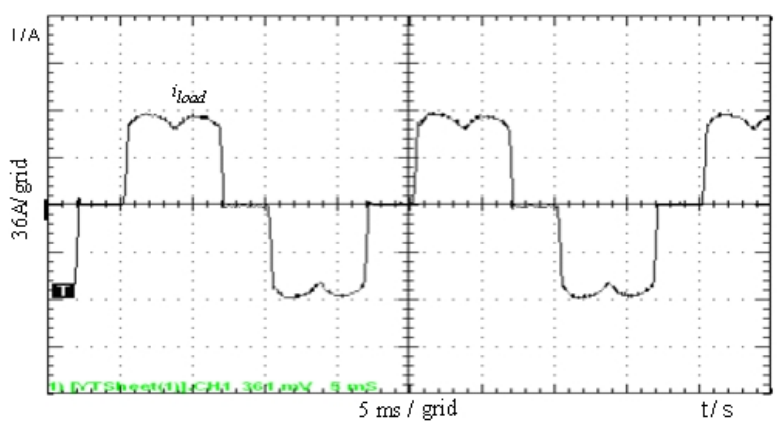

(a)

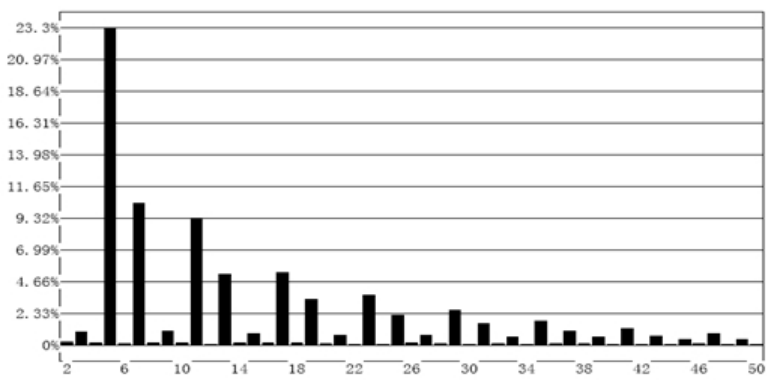

(b)

Fig. 7. (a) Steady waveform of distorted load current of one phase, (b) magnitude spectrum.

Firstly, we verify the improved selective harmonic current control in a single APF unit. The fifth order harmonic current and the seventh order harmonic current are separately compensated. The experimental results are shown in Fig.8. For the fifth order harmonic current, the 5th is reduced from $23.27 \%$ to $0.798 \%$ and the THD is correspondingly reduced to $15.934 \%$. For the seventh order harmonic current, the 7th is reduced from $10.43 \%$ to $0.525 \%$ and the THD is correspondingly reduced from $29 \%$ to $25.948 \%$. We can see that the compensation accuracy of the selective order harmonic current is significantly improved. Another important characteristic is that the nonselective harmonic currents and their harmonic distortions are unchanged. The reason is that the improved selective harmonic current control is independently implemented in a respective harmonic frame, which effectively decreases the influence on the other order harmonic currents and enhances the overall system stability. In addition, the output inductor parameters are more easily confirmed, and the different time delays caused by sampling and digitization are more easily compensated.

Secondly, the parallel strategy proposed is verified by the parallel operation of two APF units having the same capacity. Referring back to Fig.7, the effective value of the fifth harmonic current is $11.90 \mathrm{~A}$, the total effective value of the other order harmonic currents is $11.95 \mathrm{~A}$, which can be calculated from a magnitude spectrum analysis of the load current. According to the proposed parallel control strategy described above, APF unit1 compensates the fifth order harmonic current and APF unit2 compensates the other order harmonic currents. As the experimental results shown in Fig.9, the THD is reduced from $29 \%$ to $2.7 \%$ and each order harmonic current distortion is less than $1.3 \%$. The power

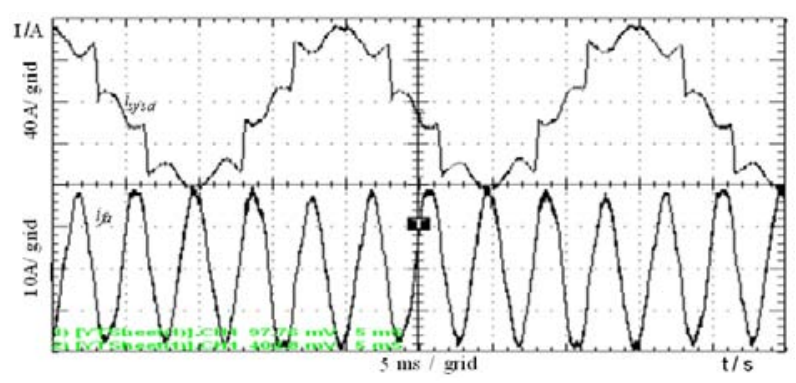

(a)

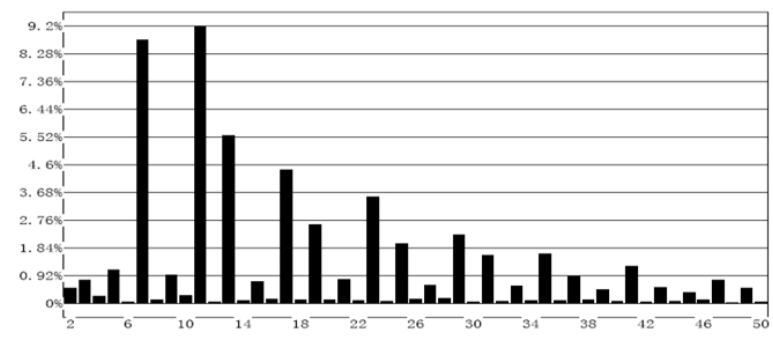

(b)

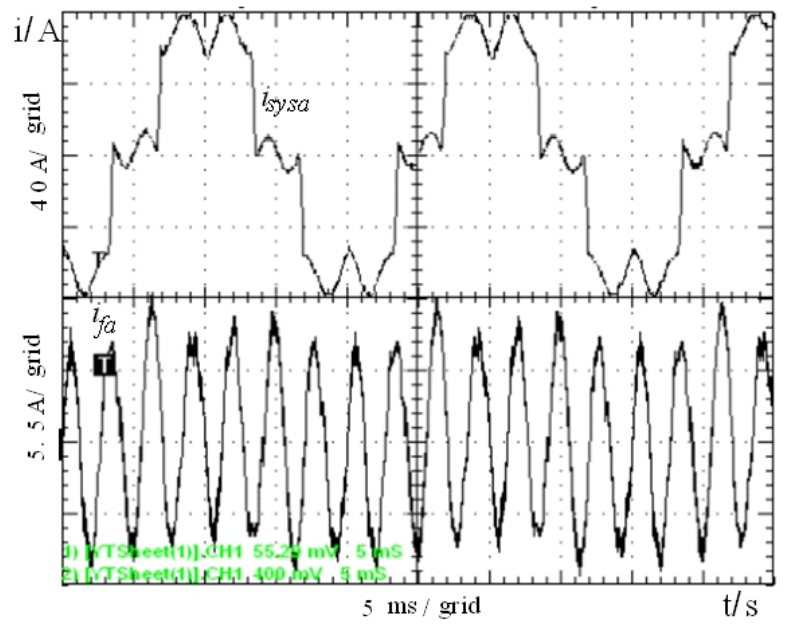

(c)

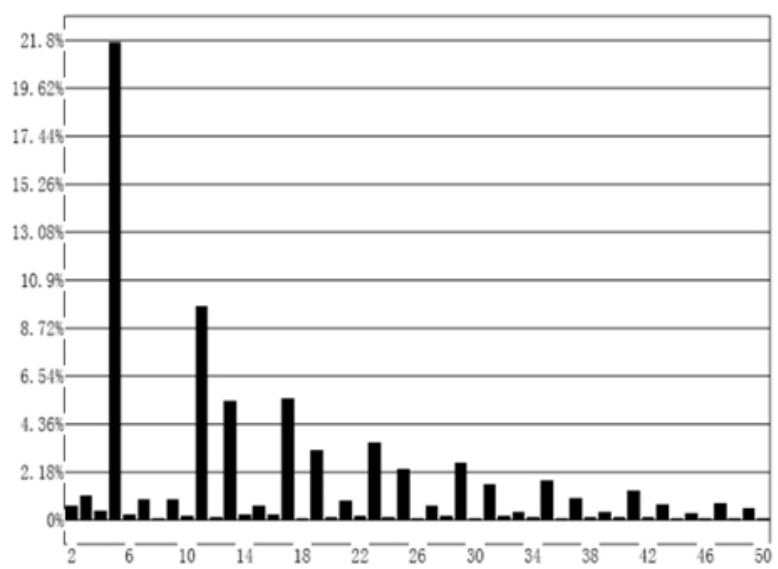

(d)

Fig. 8. (a) Power system current with only compensating the fifth order harmonic current and output current of APF, (b) magnitude spectrum, (c) Power system current with only compensating the seventh order harmonic current and output current of APF, (d) magnitude spectrum. 


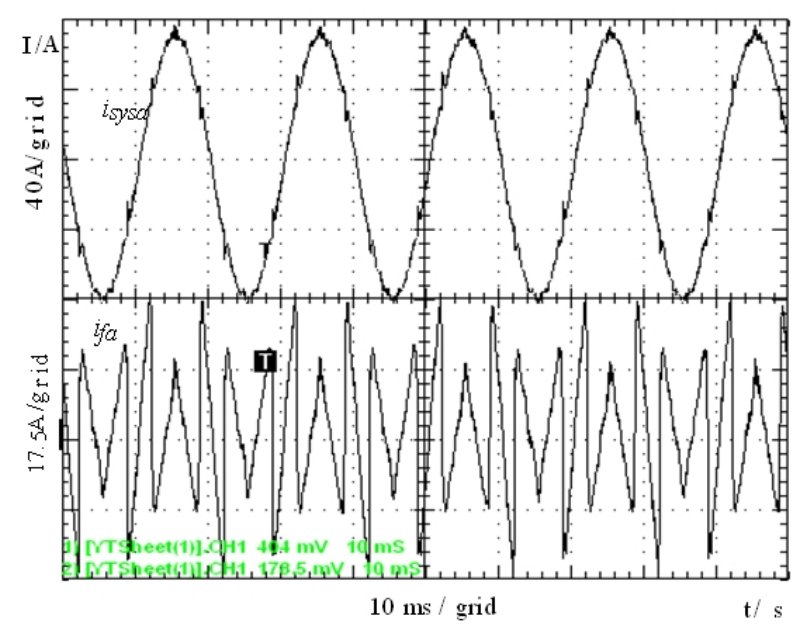

(a)

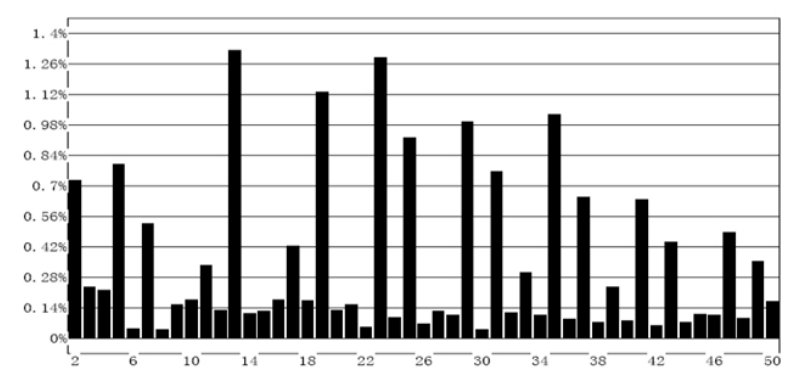

(b)

Fig. 9. (a) Compensated currents of the parallel system and output current of APF, (b) magnitude spectrum.

system current waveform quality is significantly improved. Namely, the proposed parallel control strategy can enhance the compensation accuracy in high power applications. It is necessary to explain that the strategy proposed is successfully applied where the harmonic current spectrum components do not fast change very much. Further improvement will be conducted when the load harmonic spectrum components have rapid dynamic changes.

\section{CONCLUSIONS}

The proposed parallel control strategy based on an improved selective harmonic current control in capacity proportion frequency allocation mode can achieve significant compensation accuracy. The compensated currents meet the requirement for high power applications which demand that the THD and each single order harmonic distortion are effectively reduced. Its design is explained and verified on a laboratory platform.

The most significant characteristics of the proposed parallel strategy are as follows:

- Each single harmonic distortion is obviously reduced to less than $1.3 \%$.

- The THD is significantly reduced from $29 \%$ to $2.7 \%$ and the compensated current's waveform quality is clearly rectified in the field of high power.

- A single APF unit compensates a single order harmonic current or several adjacent order harmonic currents, and thus the overall system stability is enhanced.

- Selective harmonic current control with a PI controller in the harmonic frame reduces the disturbance among the adjacent order harmonic currents.

- Straightforward installation and flexible compensation is practical, which makes it more acceptable to customers.

The proposed parallel control strategy based on an improved selective harmonic current control focuses on the application of APFs in high power applications. Both compensation accuracy and dynamic response are significantly improved.

\section{ACKNOWLEDGMENT}

The paper was supported by the National Nature Science Foundation of China (NO.50777025).

\section{REFERENCES}

[1] B. Singh, K. Al-Haddad, and A. Chandra, "A review of active filters for power quality improvement," IEEE Trans. Ind. Electron., Vol. 46, No. 5, pp. 960-971, Oct. 1999.

[2] F. Pottker and I. Barbi. "Power factor correction of non-linear loads employing a single phase active power filter: control strategy, design methodology and experimentation," IEEE Power Electronics Specialists Conference (PESC), Vol. 1, pp. 412 - 417, Jun. 1997.

[3] Ahmed. M. Massoud, Stephen. J. Finney, et al., "Three-Phase, ThreeWire, Five-Level Cascaded Shunt Active Filter for Power Conditioning, Using Two Different Space Vector Modulation Techniques," IEEE Trans. Power Del., Vol. 22, No. 4, pp.2349-2361, Oct. 2007.

[4] Z. Du, L. M. Tolbert, and J. N. Chiasson, "Active harmonic elimination for multilevel converters," IEEE Trans. Power Electron., Vol. 21, No. 2, pp. 459-469, Mar. 2006.

[5] S. J .Chiang, W. J. Ai, et al., "Parallel operation of three-phase fourwire active power filters without control interconnection," IEEE Power Electronics Specialists Conference (PESC), pp. 1202-1207, 2002.

[6] S. J .Chiang, W. J. Ai, et al., "Parallel operation of capacity-limited three-phase four-wire active power filters," IEE. Proc-Electr., Vol. 149, No.5, pp. 329-336, Sep. 2002.

[7] XueLiang Wei, et al., "Parallel control of three-phase three-wire shunt active power filters," Power Electronics and Motion Control Conference, Vol. 2, pp. 790-794, 2007.

[8] L. Asiminoaei, et al., "Performance improvement of shunt active power filter with dual parallel topology," IEEE Trans. Power Electron., Vol. 22, No. 1, pp. 247-259, JAN. 2007.

[9] Ting Qian, et al., "Parallel operation of shunt active power filters for damping of harmonic propagation in electric shipboard power system," IEEE Electric ship technologies symposium, pp. 248-254, 2005.

[10] W. M. Grady, M. J. Samotyj, and A. H. Noyola, "Survey of active power line conditioning methodologies," IEEE Trans. Power Del, Vol. 5, No. 3, pp. 1536-1542, Aug. 1990.

[11] P. Mattavelli, et al. "A closed-loop selective harmonic compensation for active filters," IEEE Trans. Ind. Appl., Vol. 37, No. 1, pp. 81-89, Jan./Feb. 2001.

[12] X. Yuan, W. Merk, H. Stemmler, and J. Allmeling, "Stationary-frame generalized integrators for current control of active power filters with zero steady-state error for current harmonics of concern under unbalanced and distorted operating conditions," IEEE Trans. Ind. Appl., Vol. 38, No. 2, pp. 523-532, Mar./Apr. 2002.

[13] P. Mattavelli and F. P. Marafao, "Repetitive-based control for selective harmonic compensation in active power filters," IEEE Trans. Ind. Electron., Vol. 51, No. 5, pp. 1018-1024, Oct. 2004.

[14] Yu Mi, et al., "A zero-steady-error control scheme of three-phase threewire active power filter," IEEE International Conference on Industrial Technology, Apr. 2008.

[15] C. Lascu, L. Asiminoaei, I. boldea, F. Blaabjerg, "High performance current controller for selective harmonic compensation in active power filter,' IEEE Trans. Power Electron., Vol. 22, No. 5, pp. 1826-1835, Sep. 2007

[16] Xie Bin, et al., "Application of moving average algorithm for shunt active power filter," Chinese power electronics, Vol. 41, No. 1, pp. 4063, Jan. 2007

[17] Hatem A. Darwish and Magdy Fikri, "Practical considerations for recursive DFT implementation in numerical relays," IEEE Trans. Power Del., Vol. 22, No. 1, pp. 42-49, Jan. 2007. 


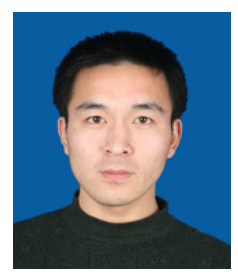

Shuquan Zhang was born in Shandong Province, China, on July 12, 1983. He received his B.E. from the China University of Petroleum, Dongying China, in 2006. He is currently pursuing his Ph.D. in the College of Electrical and Electronic Engineering, Huazhong University of Science and Technology, Wuhan, China. His current research interests include power quality improvement and power electronics applications in power systems.

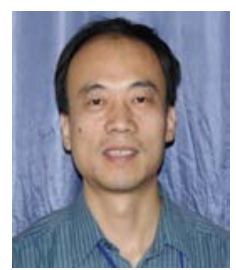

Ke Dai was born in Hubei Province, China, in 1969. He received his B.E. and Ph.D. from the Huazhong University of Science and Technology, Wuhan China, in 1990 and 2003, respectively. In 1998, he joined the Huazhong University of Science and Technology as a Lecturer and was promoted to Associate Professor in 2004. He is presently the Vice-Dean of the Department of Applied Power Electronics Engineering in the Huazhong University of Science and Technology. His current research interests include power electronics converters, electromagnetic compatibility, flexible alternating current transmission systems, custom power, and their digital control techniques.

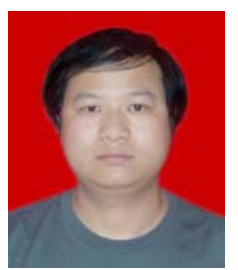

Bin Xie was born in Henan Province, China, in 1976. $\mathrm{He}$ received his B.E. from the Huazhong University of Science and Technology, Wuhan, China, in 1998, where he is currently pursuing his Ph.D. in the College of Electrical and Electronic Engineering. His current research interests include power quality improvement and power electronics applications in power systems.

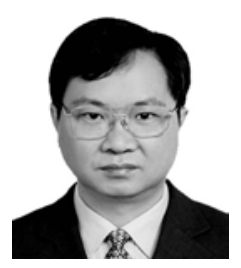

Yong Kang was born in Hubei Province, China, on October 16, 1965. He received his B.E. M.E, and Ph.D. from the Huazhong University of Science and Technology, Wuhan China, in 1988, 1991 and 1994, respectively. In 1994, he joined the Huazhong University of Science and Technology as a Lecturer and was promoted to Associate Professor in 1996 and to Full Professor in 1998. He is presently the Head of the College of Electrical and Electronic Engineering, Huazhong University of Science and Technology. He is the author of more than 60 technical papers. His current research interests include power electronics converters, AC drivers, electromagnetic compatibility, and their digital control techniques. 